\title{
Avaliação radiográfica da cobertura acetabular à cabeça femoral, após osteotomia tripla e aplicação de cunha sacroilíaca, em pelve de cadáveres de cães ${ }^{1}$
}

\author{
Everton Regonato ${ }^{2 \star}$, Júlio C. Canola ${ }^{3}$, Gilberto O. Chierice ${ }^{4}$ e João G. Padilha Filho ${ }^{3}$ \\ ABSTRACT.- Regonato E., Canola J.C., Chierice G.O. \& Padilha Filho J.G. 2009. \\ [Radiographic evaluation of acetabular covering of the head of the femur after \\ triple pelvic osteotomy and application of a sacroiliac wedge to the pelvis of canine \\ cadavers.] Avaliação radiográfica da cobertura acetabular à cabeça femoral, após \\ osteotomia tripla e aplicação de cunha sacroilíaca, em pelve de cadáveres de cães. \\ Pesquisa Veterinária Brasileira 29(8):625-631. Departamento de Clínica e Cirurgia \\ Veterinária, Faculdade de Ciência Agrárias e Veterinárias, Universidade Estadual Paulista, \\ Campus de Jaboticabal, Via de Acesso Prof. Paulo Donato Castellane s/n, Jaboticabal, \\ SP 14884-900, Brazil. E-mail: everton_unesp@yahoo.com.br \\ Attempting to avoid difficulties associated to triple pelvic osteotomy (TPO), the sacroiliac \\ wedge method was experimentally developed to increase the acetabular ventroversion. \\ The goal of this study was to apply the techniques of sacroiliac wedge and TPO to canine \\ cadavers and radiographically evaluate the efficacy of acetabular ventroversion. Ten \\ cadavers of adult dogs weighting 15-25 $\mathrm{kg}$ were used. To each right hemipelvis the \\ technique of TPO with $20^{\circ}$ and $40^{\circ}$ plates was applied. In the left hemipelvis, $20^{\circ}$ and $40^{\circ}$ \\ wedges in the sacroiliac joint were applied. Radiographic evaluations in standard \\ projections were carried out for measurement of the acetabular covering of the head of \\ the femur in the two techniques. There was no significant differences between TPO and \\ the use of sacroiliac wedge using implants of $20^{\circ}$ and $40^{\circ}$, however a significant difference \\ $(p<0,05)$ could be observed before and after application of the $20^{\circ}$ and $40^{\circ}$ implants, as \\ well as between those that received $20^{\circ}$ and of $40^{\circ}$ implants. The application of sacroiliac \\ wedge produced similar results of those observed in TPO, and it is more feasible.
}

INDEX TERMS: Diseases of dogs, surgery, hip dysplasia.

RESUMO.- Na tentativa de evitar algumas das dificuldades associadas à osteotomia pélvica tripla (OPT), foi desenvolvido experimentalmente o método de aplicação de cunha na junção sacroilíaca para aumentar a ventroversão

\footnotetext{
${ }^{1}$ Recebido em 1ㅇ de Agosto de 2008.

Aceito para publicação em 8 de março de 2009.

2 Pósgraduando em Cirurgia Veterinária, Faculdade de Ciências Agrárias e Veterinárias (FCAV), Universidade Estadual Paulista (Unesp), Campus de Jaboticabal, SP. Endereço particular: Rua Joaquim de Moraes 463, Jardim Bandeirantes, Bady Bassitt, SP 15115-000, Brasil. *Autor para correspondência: everton_unesp@yahoo.com.br

2 Departamento de Clínica e Cirurgia Veterinária, FCAV, Unesp, Campus de Jaboticabal, Via de Acesso Prof. Paulo Donato Castellane s/n, Jaboticabal, SP 14884-900, Brasil.

${ }^{3}$ Departamento de Química e Física Molecular, Instituto de Química de São Carlos, Universidade de São Paulo, Av. Trabalhador SãoCarlense 400, São Carlos, SP 13560-970, Brasil.
}

acetabular. O objetivo deste estudo foi aplicar as técnicas de cunha sacroilíaca e OPT em cadáveres e avaliar radiograficamente a eficácia da ventroversão acetabular. Para tal, foram utilizados 10 cadáveres de cães, adultos, com $15-25 \mathrm{~kg}$. Em cada hemipelve direita foi realizada OPT com placas de $20^{\circ}$ e $40^{\circ}$. Na hemipelve esquerda foram aplicadas cunhas nas articulações sacroilíacas de $20^{\circ}$ e $40^{\circ}$. Avaliações radiográficas em projeções ventrodorsais foram realizadas para mensuração da cobertura acetabular à cabeça femoral nas duas técnicas. De acordo com os dados obtidos pode-se observar que não houve diferença entre a técnica de OPT e o uso de cunha sacroilíaca utilizando implantes de $20^{\circ}$ e $40^{\circ}$, mas ocorreu diferença significativa $(p<0,05)$ entre os cães antes e após a aplicação dos implantes de $20^{\circ}$ e $40^{\circ}$, e também entre os que receberam implantes de $20^{\circ}$ e os de $40^{\circ}$. A aplicação de cunha sacroilíaca produziu resulta- 
dos semelhantes à OPT, e também se mostrou como de mais fácil aplicação.

TERMOS DE INDEXAÇÃO: Doenças de cães, cirurgia, displasia coxofemoral.

\section{INTRODUÇÃO}

Para o tratamento da displasia coxofemoral podem ser adotados métodos conservativos, clínicos e cirúrgicos. Diversos procedimentos cirúrgicos podem ser realizados com o objetivo de impedir a progressão da doença articular degenerativa. No entanto, a depender do estágio em que se encontra a articulação do paciente, a técnica cirúrgica empregada pode variar (Padilha Filho 1992).

A osteotomia pélvica tripla (OPT) é indicada para cães jovens com sinais clínicos de displasia coxofemoral e que demonstram sinais de instabilidade da articulação coxofemoral. A OPT é um método preventivo e terapêutico para displasia coxofemoral, especialmente para cães jovens de raças grandes e gigantes, entre cinco e 12 meses de idade (David \& Kasper 1992). Incongruência grave entre a cabeça femoral e o acetábulo, artrite deformante da articulação do quadril, acetábulo raso e atrofia muscular da pelve e coxa, são contra-indicações para OPT, assim como desordens metabólicas e neurológicas. O procedimento fornece rotação lateral do eixo axial do acetábulo para estabilizar a cabeça femoral dentro do acetábulo em posição funcional (Slocum \& Devine 1987, Slocum \& Devine 1992). O grau de rotação deve ser suficiente para permitir adequada cobertura à cabeça femoral. Rotação insuficiente do acetábulo pode resultar em contínua instabilidade e progressão da doença articular degenerativa (Tomlinson \& Cook 2002). A rotação considerada ideal ainda não foi determinada; contudo, em geral se aceita que a rotação excessiva compromete a extensão e abdução do membro pélvico, por promover a colisão do colo femoral contra a borda acetabular (Schrader 1981, Slocum \& Devine 1986). Segundo alguns autores (Tomlinson \& Cook 2002), a rotação de $20^{\circ}$ do acetábulo fornece cobertura apropriada à cabeça femoral, ao mesmo tempo em que evita a rotação excessiva, enquanto outros (Slocum \& Devine 1987) afirmam que a rotação acetabular de $20^{\circ}$ a $30^{\circ}$ é adequada para a maioria dos casos, exceto para os cães com subluxação.

As complicações associadas à OPT incluem o estreitamento excessivo do canal pélvico, constipação, lesão do nervo isquiático, falha dos implantes, afrouxamento dos parafusos, incongruência persistente entre a cabeça femoral e o acetábulo, infecções e problemas na incisão cirúrgica (Maclaughlin Jr. \& Tomlinson 1996). O aumento na profundidade dos parafusos dentro do sacro no aspecto cranial da osteotomia ilial, juntamente com a cerclagem na osteotomia isquial, ajudam a evitar a migração dos parafusos (Simmons et al. 2001).

A utilização de cunhas na junção sacroilíaca com intuito de mimetizar o efeito da OPT foi sugerida com a finalidade de promover rotação axial do acetábulo (Conze- mius et al. 1999). Esta técnica propicia a realização da ventroversão acetabular bilateral, já que o cão é posicionado em decúbito esternal, possibilitando o acesso a ambas articulações sacroilíacas, através de única incisão na linha média dorsal do sacro. Este estudo tem como objetivo aplicar as técnicas de cunha sacroilíaca e OPT em cadáveres e avaliar radiograficamente a eficácia da ventroversão acetabular.

\section{MATERIAL E MÉTODOS}

Foram utilizados dez cadáveres de cães, adultos, de porte médio a grande (peso corporal de 15-25 kg), isentos de displasia coxofemoral aos exames do ângulo de Norberg e porcentagem de cobertura acetabular. No acetábulo direito foi aplicada a técnica de OPT com placas de poliuretana de mamona (Ricinus communis) de $20^{\circ} \mathrm{e}$, em seguida, de $40^{\circ}$. Para se obter a ventroversão acetabular contralateral foram utilizadas cunhas do mesmo material com ângulos de $20^{\circ}$ e $40^{\circ}$ nas junções sacroilíacas (Fig.1). As placas foram confeccionadas com poliuretana compacta, para maior resistência, e as cunhas produzidas com bloco poroso, com intuito de melhorar sua aderência aos ossos sacro e ílio.

Para a OPT foi utilizada a modificação (Tarvin \& Lenehan 1996) de uma técnica anteriormente descrita (Slocum \& Devine 1986). O grau de rotação acetabular foi determinado pela angulação da placa empregada a cada momento do procedimento. Foram utilizadas placas de $20^{\circ}$ e $40^{\circ}$ sem orifícios, que

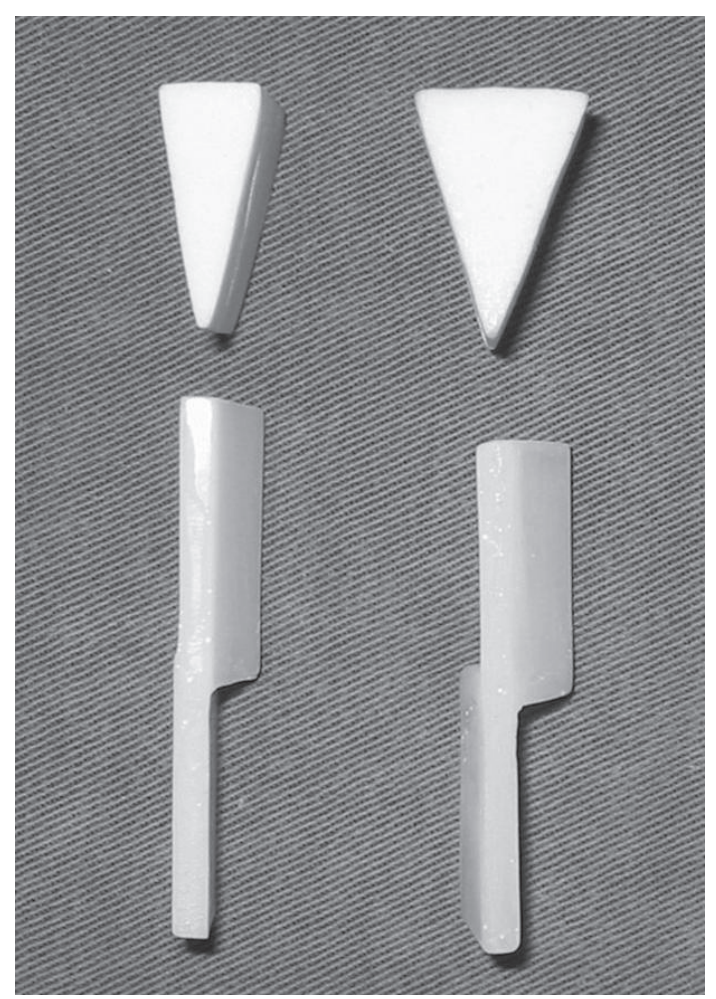

Fig.1. Na parte superior da figura estão as cunhas de poliuretana de mamona (Ricinus communis), com ângulos de $20^{\circ}$ e $40^{\circ}$, da esquerda para a direita respectivamente. Na parte inferior da figura, as placas para osteotomia pélvica tripla de mesmo material e ângulos das cunhas, da esquerda para a direita respectivamente. 


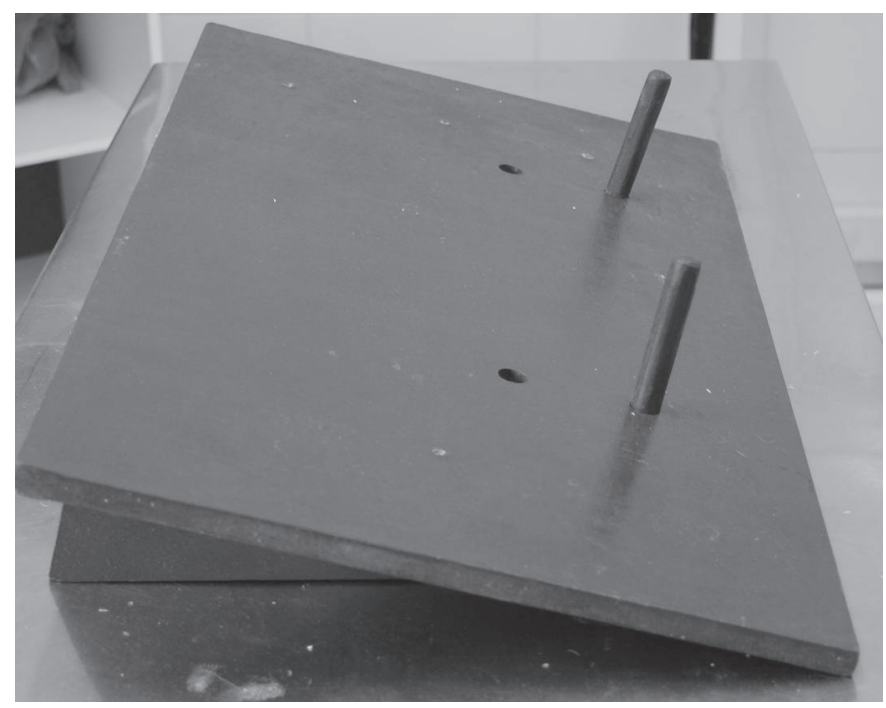

Fig.2. Dispositivo usado para posicionar os cães em decúbito lateral com inclinação entre $30^{\circ}$ e $45^{\circ}$ graus, para realização da técnica de cunha sacroilíaca.

foram perfurados durante o procedimento cirúrgico. Os orifícios nas placas foram realizados aleatoriamente, considerando a adaptação do implante no osso, evitando-se os previamente produzidos. Inicialmente, a placa de $20^{\circ}$ foi utilizada para promover ventroversão e estabilização do acetábulo direito que, após avaliação radiográfica, foi substituída pela de $40^{\circ}$, passando também por acompanhamento radiográfico.

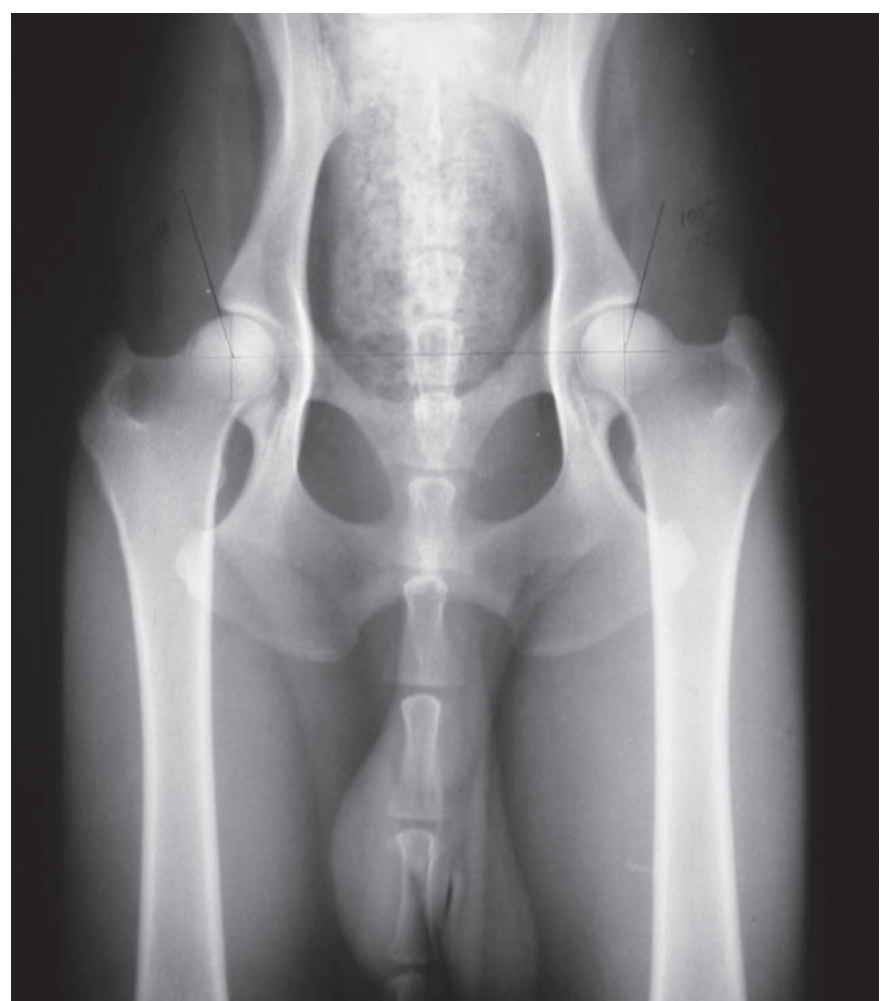

Fig.3. Imagem radiográfica ventrodorsal padrão no período préoperatório em cadáver de cão, evidenciando o método do ângulo de Norberg usado para avaliação da cobertura acetabular à cabeça femoral.

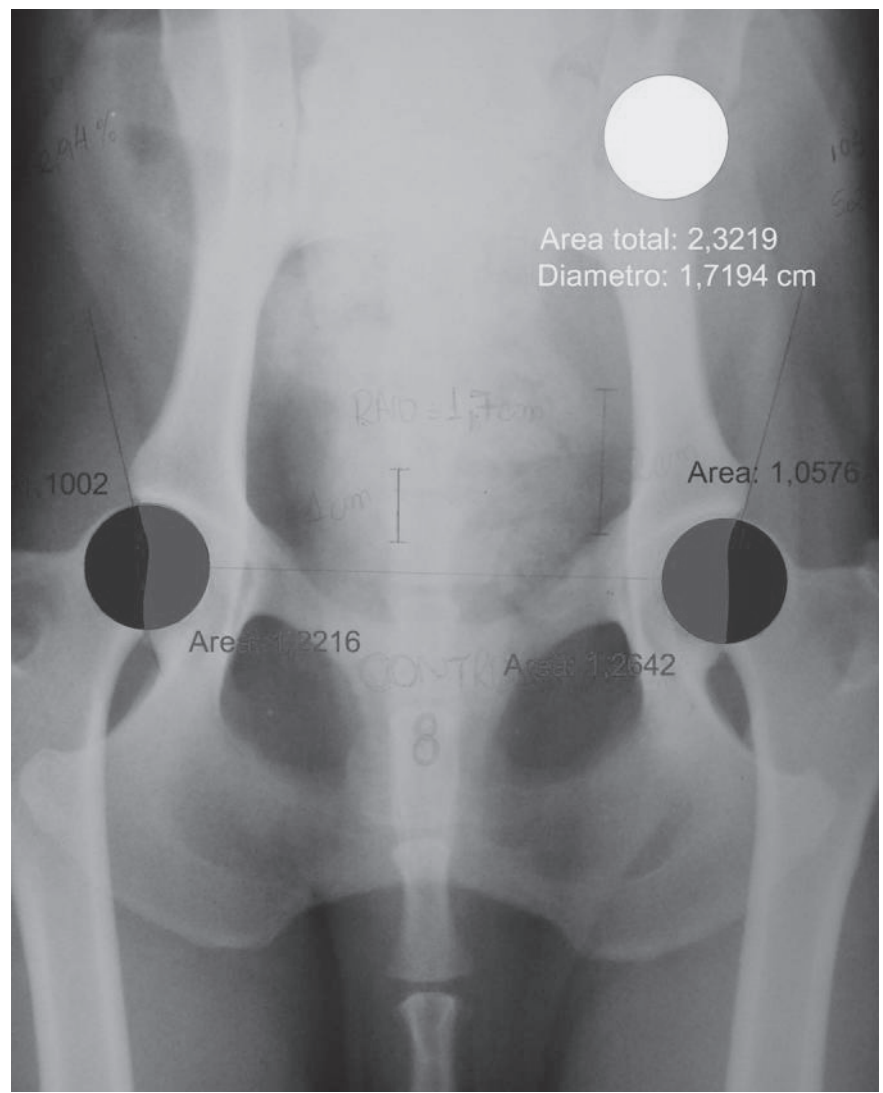

Fig.4. Imagem radiográfica ventrodorsal no período pré-operatório em cadáver de cão, evidenciando a avaliação através da Porcentagem de Cobertura Acetabular usando o programa AutoCAD ${ }^{\circledR} 2007$ Autodesk para avaliação da cobertura acetabular à cabeça femoral. A área vermelha representa a cabeça femoral coberta pelo acetábulo e a azul mais a vermelha representam a área total da cabeça femoral.

A aplicação da cunha na junção sacroilíaca foi realizada conforme técnica descrita anteriormente (Conzemius et al. 1999). As cunhas possuíam dimensões de $2,5 \mathrm{~cm}$ de altura (h), $3 \mathrm{~cm}$ de comprimento em angulação (a) de $20^{\circ}$ e $40^{\circ}$. Procederam-se osteotomias no púbis e ísquio da mesma forma da OPT. A abordagem ao osso sacro foi realizada semelhante ao descrito anteriormente (Piermattei \& Johnson 2004). O cadáver do cão foi posicionado em decúbito lateral direito, inclinado entre $30^{\circ}$ e $45^{\circ} \mathrm{com}$ auxílio de suporte previamente projetado (Fig.2); para a aplicação da cunha, foram seccionados os ligamentos sacroilíacos dorsais e abertura da junção sacroilíaca. Ato contínuo, a cunha foi introduzida na articulação fibrocartilaginosa sacroilíaca, com a base triangular nivelando-se com o plano da lâmina dorsal do sacro. Procedeu-se perfuração de dois orifícios de $3 \mathrm{~mm}$, da superfície lateral da asa ilíaca, transpassando a cunha e perfurando o sacro. Em seguida, dois parafusos de aço inoxidável 304 de 4,2mm de diâmetro e 50mm de comprimento, foram usados para fixar o ílio, cunha e sacro. As cunhas foram aplicadas na hemipelve esquerda de cada cadáver, sendo substituídas à medida que se processava a avaliação radiográfica.

Foi adotado o posicionamento ventrodorsal padronizado pela Orthopaedic Foundation for Animals para a avaliação radiográfica. Utilizaram-se dois métodos de avaliação da cobertura ace- 
tabular: o método do ângulo de Norberg (Fig.3) e a porcentagem de cobertura acetabular. A mensuração da porcentagem de cobertura acetabular foi realizada com o programa AutoCAD ${ }^{\circledR}$ 2007 (Autodesk, 111 Mclnnis Parkway, San Rafael, CA, USA) medindo-se a área total da cabeça femoral e a sua porção coberta pelo acetábulo (Fig.4). Ambos foram aplicados e mensurados pré-operatoriamente e após o uso dos implantes de $20^{\circ}$ e $40^{\circ}$.

Foi realizada análise das médias dos ângulos de Norberg e médias de porcentagem de cobertura acetabular no período pré-operatório e após aplicação dos implantes de $20^{\circ}$ e $40^{\circ}$, utilizando-se o Teste t de Student, com nível de significância de $5 \%$.

\section{RESULTADOS E DISCUSSÃO}

Pela mensuração da porcentagem de cobertura acetabular, as médias dos cadáveres caninos que receberam implantes (placas e cunhas) de mesmo ângulo não apresentaram diferença significativa entre o lado direito e o esquerdo, quando comparado ao período pré-operatório, evidenciando a similaridade entre a OPT e o uso de cunha sacroilíaca. Desta forma, após aplicação dos implantes de $20^{\circ}$ e $40^{\circ}(p<0,05)$, houve ventroversão acetabular de forma semelhante.

Na mensuração do ângulo de Norberg, não houve diferença significativa entre as técnicas utilizadas. A comparação entre o período pré-operatório e os cadáveres de cães submetidos a implantes de $20^{\circ}$ e $40^{\circ}$ mostrou diferença significativa $(p>0,05)$ tanto no uso das placas quanto das cunhas, evidenciando a ocorrência da ventroversão após os procedimentos cirúrgicos. Entre os cadáveres do grupo tratado com implantes de $20^{\circ}$ e os de $40^{\circ}$ também ocorreu diferença, com maior cobertura acetabular da cabeça femoral com implantes de $40^{\circ}$. Esses resultados foram observados tanto pela mensuração da porcentagem de cobertura acetabular quanto pela mensuração do ângulo de Norberg, fornecendo dados que apresentavam forte correlação entre os métodos (Quadros 1 e 2).

Numa investigação da prevalência da displasia coxofemoral (Rettenmaier et al. 2002) foi mencionada a estreita relação entre a porcentagem de cobertura acetabular e o ângulo de Norberg, que foram semelhantes aos resultados obtidos nesse estudo. Esses autores informam que 0

\begin{tabular}{|c|c|c|}
\hline \multirow[t]{2}{*}{ Período } & \multicolumn{2}{|c|}{ Porcentagem de cobertura acetabular } \\
\hline & $\begin{array}{l}\text { Hemipelve direita } \\
\left(\text { (OPTa) }^{\mathrm{a}}\right)\end{array}$ & $\begin{array}{c}\text { Hemipelve esquerda } \\
\text { (cunha sacroilíaca) }\end{array}$ \\
\hline Período pré-operatório & $56,44^{*}$ a $A$ & 54,35 a $A$ \\
\hline $20^{o b}$ & 81,65 b A & 75,49 b A \\
\hline $40^{\circ \mathrm{C}}$ & 92,97 с A & 87,89 с A \\
\hline
\end{tabular}

a OPT: osteotomia pélvica tripla; ${ }^{\mathrm{b}}$ implantes com ângulos de $20^{\circ}$ graus; ${ }^{\mathrm{C}}$ implantes com ângulos de $40^{\circ}$ graus. *Médias seguidas da mesma letra minúscula na coluna e maiúscula na linha não diferem entre si pelo teste $\mathrm{T}$ de Student.
Quadro 2. Médias dos cães no período pré e pós-operatório, empregando o método do ângulo de Norberg para mensuração da ventroversão acetabular e análise estatística através do Teste $t$ de Student, a $5 \%$ de significância

\begin{tabular}{lcc}
\hline \multirow{2}{*}{ Período } & \multicolumn{2}{c}{ Ângulo de Norberg } \\
\cline { 2 - 3 } & $\begin{array}{c}\text { Hemipelve direita } \\
\text { (OPTa) }\end{array}$ & $\begin{array}{c}\text { Hemipelve esquerda } \\
\text { (cunha sacroilíaca) }\end{array}$ \\
\hline Período pré-operatório & $108^{*}$ a A & 107,3 a A \\
$20^{\circ \mathrm{b}}$ & $123,1 \mathrm{~b} \mathrm{~A}$ & $119,6 \mathrm{~b} \mathrm{~A}$ \\
$40^{\circ \mathrm{c}}$ & $137,6 \mathrm{c} \mathrm{A}$ & $137,2 \mathrm{c} \mathrm{A}$ \\
\hline
\end{tabular}

$\bar{a}$ OPT: osteotomia pélvica tripla; ${ }^{b}$ implantes com ângulos de $20^{\circ}$ graus; ${ }^{C}$ implantes com ângulos de $40^{\circ}$ graus. *Médias seguidas da mesma letra minúscula na coluna e maiúscula na linha não diferem entre si pelo teste $\mathrm{T}$ de Student.

escore da Orthopaedic Foundation for Animals também mantém correlação com a porcentagem de cobertura acetabular e o ângulo de Norberg. Outros autores (Tomlinson \& Johnson 2000) também evidenciaram a correlação entre esses métodos de avaliação radiográfica da displasia coxofemoral, sugerindo que o ângulo de Norberg tem proporcionalidade com a porcentagem de cobertura acetabular. Já outros autores afirmam que utilizando $105^{\circ}$ para diferenciar cães normais e displásicos pode gerar resultados falso-positivos ou falso-negativos. Cada raça possui valores específicos que os distinguem entre normais e displásicos (Tomlinson \& Johnson 2000, Culp et al. 2006). Com o avanço da idade a gravidade da displasia coxofemoral aumenta (Lust et al. 1993) produzindo também menores valores para o ângulo de Norberg e porcentagem de cobertura acetabular. Estes fatos tornam importante a padronização das idades e raças para avaliação da cobertura acetabular.

A razão para o uso dos implantes em poliuretana de mamona foi a facilidade de moldagem e biocompatibilidade. Além disso, este experimento serviu de modelo experimental para futuros estudos de resistência desses materiais e suas aplicações clínicas. As placas para OPT não apresentavam orifícios prévios para colocação dos parafusos, tornando-as mais versáteis e fáceis de serem utilizadas, embora apresentaram fragilidade no ponto de angulação favorecendo a quebra do implante. Foram efetivamente empregadas placas e cunhas com ângulos de 20 e $40^{\circ}$, semelhantes aos ângulos das placas para osteotomias pélvicas caninas anteriormente (Slocum \& Devine 1986). As placas possuíam dimensões de $60 \mathrm{~mm}$ de comprimento, $17 \mathrm{~mm}$ de largura e $4 \mathrm{~mm}$ de espessura, diferindo em sua conformação e tamanho das placas usualmente recomendadas como a placa de compressão dinâmica e placa para osteotomia pélvica canina (Slocum \& Devine 1986, Slocum \& Devine 1987, Hunt \& Litsky 1988, Tarvin \& Lenehan 1996). Os implantes com angulações maiores (placas e cunhas) poderiam produzir rotação excessiva do acetábulo, comprometendo a abdução dos membros, diminuindo o limite de movimentação da articulação (Schrader 1986, Slocum \& Devine 1986, Slocum \& Devine 1987). 


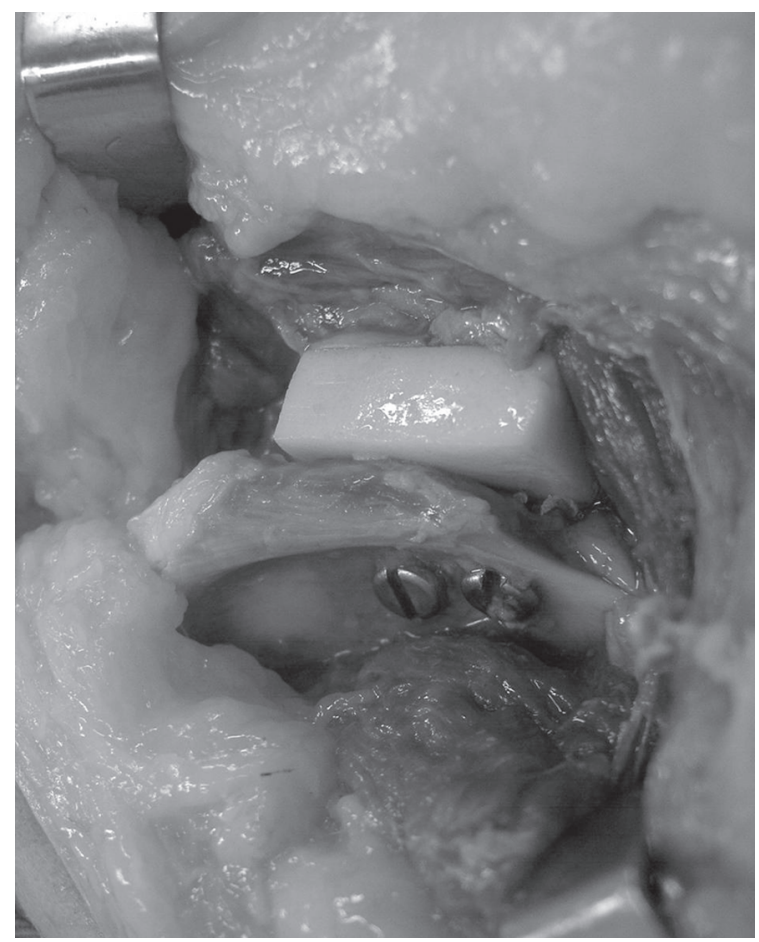

Fig.5. Imagem radiográfica evidenciando a posição da cunha de poliuretana de mamona na junção sacroilíaca, em cão submetido à aplicação de cunha sacroilíaca. Houve boa coaptação na junção após inserção de dois parafusos desde a porção lateral da crista ilíaca atravessando a cunha e fixando no sacro.

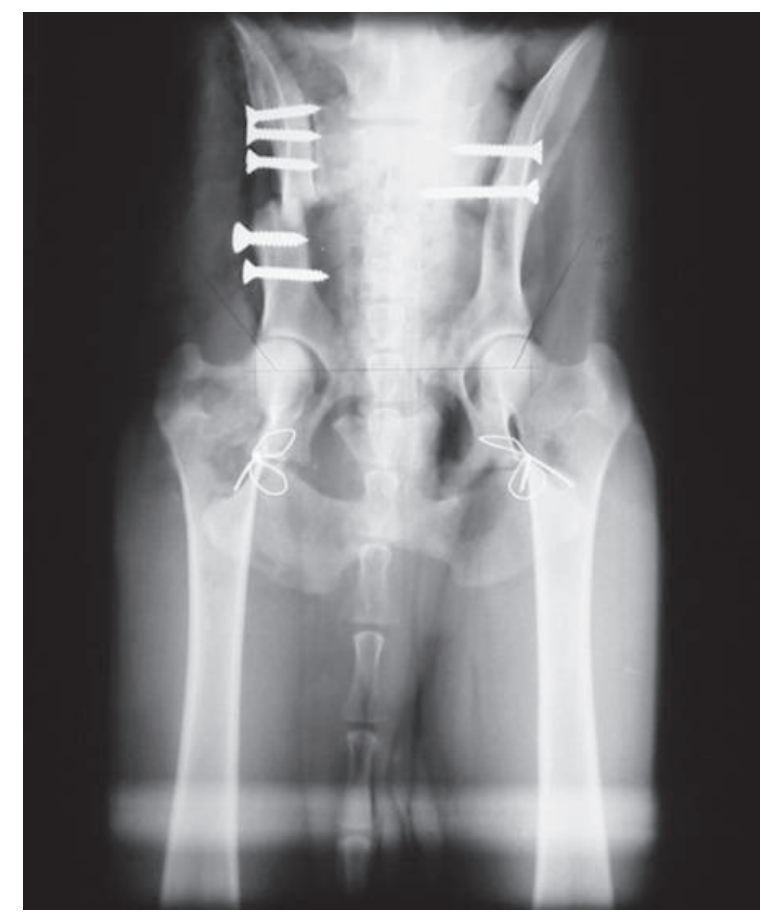

Fig.6. Imagem radiográfica do período pós-operatório evidenciando o aumento na cobertura acetabular após as técnicas de osteotomia pélvica tripla e cunha sacroilíaca em cadáver de cão. As placas e cunhas não podem ser vistas na imagem radiográfica por se tratar de material radioluscente.
Quanto à angulação das placas, há relatos indicando que a rotação acetabular de $20^{\circ}$ é suficiente para promover estabilidade à articulação coxofemoral (Tomlinson \& Cook 2002), enquanto outros autores (Rasmussen et al. 1998) afirmaram que $45^{\circ}$ é a quantidade suficiente de rotação para alcançar estabilidade da articulação coxofemoral displásica, independente da mensuração do ângulo de Norberg e porcentagem de cobertura acetabular antes da cirurgia. Esses relatos contribuíram para que fossem utilizados apenas implantes de $20^{\circ}$ e $40^{\circ}$.

As cunhas de $20^{\circ}$ e $40^{\circ}$ adaptaram-se bem na junção sacroilíaca, após serem fixadas com dois parafusos de aço inoxidável 304 de $4,2 \mathrm{~mm}$ de espessura e $50 \mathrm{~mm}$ de comprimento (Fig.5). Estes parafusos são biocompatíveis e resistentes à corrosão, podendo ser empregados na Veterinária sem riscos à saúde dos animais (Costa 2003), além de mostrarem boa fixação e adaptação ao osso. As placas e cunhas não foram vistas nas imagens radiográficas por se tratarem de material radiolucente, sendo observados apenas os parafusos fixando as mesmas após as osteotomias (Fig.6).

O uso de dois parafusos longos para a estabilização da osteotomia do ílio na OPT penetrando profundamente no sacro e não adentrando a canal medular, minimiza o afrouxamento dos mesmos (Simmons et al. 2001). A colocação erroneamente ventral ao sacro pode resultar em lesão do nervo isquiático (Whelan et al. 2004). Em virtude destas observações, foram utilizados dois parafusos de $50 \mathrm{~mm}$ de comprimento e 4,2 $\mathrm{mm}$ de espessura para estabilização da cunha na junção sacroilíaca, produzindo boa coaptação na avaliação radiográfica pós-operatória. Alguns autores (Planté et al. 1997) utilizaram parafusos esponjosos de 4,0mm para estabilização das osteotomias, os quais promoveram boa fixação inicial das placas em animais jovens; esse relato pode contribuir para futuro estudo in vivo, ao se utilizar parafusos semelhantes e igualmente longos para estabilização das cunhas na junção sacroilíaca.

Quando se utilizou a porcentagem de cobertura acetabular para avaliação, observou-se que através da comparação das médias dos resultados, não houve diferença significativa entre o lado direito e esquerdo da pelve dos cães no período pré-operatório. Após a realização das cirurgias com implantes de $20^{\circ}$ e $40^{\circ}$, a mesma avaliação entre as médias revelou que não houve diferença significativa entre o lado direito e esquerdo, evidenciando que a OPT e a aplicação da cunha sacroilíaca produzem resultados semelhantes. Já a comparação entre as médias dos cães antes e após aplicação de implantes $20^{\circ}$ mostrou diferença significativa, o que sugere melhora na cobertura acetabular. Nos cães que receberam implantes de $40^{\circ}$, comparando-se as duas técnicas, não houve diferença significativa, mas em relação ao período pré-operatório e os que receberam implantes de $20^{\circ}$ ocorreu diferença significativa, melhorando a cobertura acetabular.

Os mesmos resultados anteriormente descritos aplicam-se na avaliação do ângulo de Norberg, apresentan- 
do forte semelhança entre os resultados observados em cada método de avaliação, corroborando com observações anteriores (Tomlinson \& Johnson 2000).

É relatado que independentemente da técnica utilizada para correção da displasia coxofemoral, a porcentagem de cobertura acetabular e o ângulo de Norberg são importantes para avaliar o objetivo imediato que é a rotação axial lateral do acetábulo (Rasmussen et al. 1998). Assim, neste estudo, a rotação do acetábulo foi observada tanto na mensuração do ângulo de Norberg quanto na porcentagem de cobertura acetabular.

Pelo fato da aplicação da cunha sacroilíaca poder ser realizada bilateralmente no mesmo procedimento cirúrgico, torna-se necessária a liberação de ambos os segmentos acetabulares para rotação axial com mínimo comprometimento da arquitetura pélvica. Com base em experimentos anteriores (Borostyankoi et al. 2003) é possível que se realizando apenas ostectomias bilaterais do púbis, poder-se-iam liberar os segmentos acetabulares. Esses autores realizaram OPT bilateralmente utilizando apenas três osteotomias (duas nos corpos ilíacos e outra na sínfise pélvica); a osteotomia do púbis foi realizada retirando-se uma cunha, com limites de $5 \mathrm{~mm}$ de cada lado da sínfise púbica na porção cranial do púbis, que se estendeu até a $5 \mathrm{~mm}$ cranialmente à borda cranial do forâmen obturador. Para a aplicação da cunha sacroilíaca este acesso ao púbis poderia ser adotado, minimizando a modificação da arquitetura pélvica. Por outro lado há pesquisadores (Conzemius et al. 1999) que acreditam que em cães jovens a osteotomia talvez não seja necessária devido à maleabilidade da sínfise púbica.

Os acessos cirúrgicos e a técnica de aplicação da cunha sacroilíaca mostraram-se de baixa complexidade, em comparação à OPT. Foram empregados três acessos cirúrgicos para a aplicação da cunha na junção sacroilíaca, com acessos ao púbis e ísquio semelhantes à OPT conforme descritos anteriormente (Tarvin \& Lenehan 1996) e outro acesso à junção sacroilíaca com o cadáver do cão posicionado em decúbito lateral direito inclinado entre $30^{\circ}$ a $45^{\circ}$ utilizando, para tal, suporte previamente projetado; essa abordagem difere do preconizado por outros autores (Conzemius et al. 1999) que sugerem que o cão seja posicionado em decúbito esternal. O afastamento lateral do músculo glúteo médio foi importante para a aplicação dos parafusos na estabilização da cunha. $O$ ato cirúrgico foi mais dinâmico comparado à OPT realizada na hemipelve contralateral.

Ao contrário de estudos anteriores (Conzemius et al. 1999), em que se realizaram projeções radiográficas apenas com a peça anatômica da pelve numa posição perpendicular à mesa e no sentido craniocaudal, este estudo foi realizado com o cadáver do cão em decúbito dorsal, na posição ventrodorsal padrão. No estudo mencionado anteriormente (Conzemius et al. 1999), foi realizado um estudo biomecânico em peças de pelve dotadas de pinos de aço, colocados nas bordas acetabulares e outro passando pelo centro do sacro e sínfise púbica. A rotação do acetábulo era mensurada pelo ângulo formado entre o pino sacral e o pino da borda acetabular após a aplicação de cada implante. No presente estudo, a aplicação dos implantes foi feita em cadáveres e as mensurações realizadas pelo ângulo de Norberg e pela porcentagem de cobertura acetabular e os resultados foram praticamente os mesmos.

Estudos in vivo devem ser realizados para comprovar o efeito e a eficiência da cunha sacroilíaca na correção da displasia coxofemoral.

\section{CONCLUSÕES}

A técnica cirúrgica empregada para aplicação da cunha sacroilíaca é simples, permite boa visibilidade das estruturas anatômicas, não apresenta dificuldades para sua realização e o ato é mais dinâmico comparado a OPT.

A avaliação através do ângulo de Norberg e a porcentagem de cobertura acetabular proporcionam boa avaliação da cobertura acetabular.

A utilização de cunhas em poliuretana de mamona permite fácil aplicação dos parafusos e proporciona boa coaptação à junção sacroilíaca.

Houve aumento significativo da ventroversão com ambas as técnicas avaliadas, que foi proporcional a angulação dos implantes utilizados; os resultados obtidos são animadores.

Agradecimentos.- À Fundação de Amparo à Pesquisa do Estado de São Paulo (FAPESP), pela concessão da bolsa de estudos, e à empresa Poliquil Araraquara Polímeros Químicos Ltda, pelo apoio essencial para a realização do projeto de pesquisa.

\section{REFERÊNCIAS}

Borostyankoi F., Rooks R.L., Kobluk C.N., Reed A.L. \& Littledike E.T. 2003. Results of single-session bilateral triple pelvic osteotomy with an eight-hole iliac bone plate in dogs: 95 cases (1996-1999). J. Am. Vet. Med. Assoc. 222:54-59.

Conzemius M.G., Aper R.L. \& Brown M.D. 1999. Evaluation of sacroiliac wedge rotation to increase acetabular ventroversion: A canine cadaver study. Vet. Comp. Orthop. Traumotol. 12:173-177.

Costa J.L.O. 2003. Acetabuloplastia extracapsular em cães com cartilagem auricular de bovino conservada com glicerina. Tese de Doutorado em Cirurgia Veterinária, Faculdade de Ciências Agrárias e Veterinárias, Universidade Estadual Paulista, Jaboticabal, SP. 84p.

Culp W.T.N., Kapatkin A.S., Gregor T.P., Powers M.Y., Mckelvie P.J. \& Smith G.K. 2006. Evaluation of the Norberg angle threshold: A comparison of Norberg angle and distraction index as measurements of degenerative joint disease susceptibility in seven breeds of dogs. Vet. Surg. 36:453-459.

David T.H. \& Kasper M. 1992. Triple pelvic osteotomy with axial acetabular rotation in canine hip dysplasia. Eur. J. Companion Anim. Pract. 2:23-38.

Hunt C.A. \& Litsky A.S. 1988. Stabilization of canine pelvic osteotomies with AO/ASIF plates and screws. Vet. Comp. Orthop. Traumatol. 1:5257.

Lust G., Williams A.J., Burton-Wurster N., Pijanowski G.J., Beck K.A., Rubin G. \& Smith G.K. 1993. Joint laxity and its association with hip dysplasia in Labrador Retrievers. Am. J. Vet. Res. 54:1990-1999.

MacLaughlin Jr R. \& Tomlinson J. 1996. Treating canine hip dysplasia with triple pelvic osteotomy. Vet. Med. 96:126-136.

Padilha Filho J.G. 1992. Patologia da bacia e articulação coxofemoral, 
p.15-26. In: Daleck C.R., Baptista L.C. \& Mukai L.S. (Eds), Tópicos em Cirurgia de Cães e Gatos. Funep-Unesp, Jaboticabal.

Piermattei D.L. \& Johnson K.A. 2004. An Atlas of Surgical Approaches to the Bones and Joints of the Dog and Cat. 4th ed. W.B. Saunders, Philadelphia.

Planté J., Dupuis J., Beauregard G., Bonneau N.H. \& Breton L. 1997. Long-term results of conservative treatment, excision arthroplasty and triple pelvic osteotomy for the treatment of hip dysplasia in the immature dog. Part 1. Radiographic and physical results. Vet. Comp. Orthop. Traumatol. 10:101-110.

Rasmussen L.M., Kramek B.A. \& Lipowitz A.J. 1998. Preoperative variables affecting long-term outcome of triple pelvic osteotomy for treatment of naturally developing hip dysplasia in dogs. J. Am. Vet. Med. Assoc. 213:80-85.

Rettenmaier J.L., Keller G.G., Latimer J.C., Corley E.A. \& Ellersieck M.R. 2002. Prevalence of canine hip dysplasia in a veterinary teaching hospital population. Vet. Radiol. Ultrasound 43:313-318.

Schrader S.C. 1981. Triple pelvic osteotomy of the pelvis as a treatment for canine hip dysplasia. J. Am. Vet. Med. Assoc. 178:39-44.

Schrader S.C. 1986. Triple osteotomy of the pelvis and trochanteric osteotomy as a treatment for hip dysplasia in the immature dog: The surgical technique and results of 77 consecutive operations. J. Am. Vet. Med. Assoc. 189:659-665.
Simmons S., Johnson A.L. \& Schaeffer A.L. 2001. Risk factors for screw migration after triple pelvic osteotomy. J. Am. Anim. Hosp. Assoc. 37:269-273.

Slocum B. \& Devine T. 1986. Pelvic osteotomy technique for axial rotation of the acetabular segment in dogs. J. Am. Anim. Hosp. Assoc. 22:331338.

Slocum B. \& Devine T. 1987. Pelvic osteotomy in the dog as treatment for hip dysplasia. Semin. Vet. Med. Surg. Small Anim. 2:107-116.

Slocum B. \& Devine T.D. 1992. Pelvic osteotomy for axial rotation of the acetabular segment in dogs with hip dysplasia. Vet. Clin. North Am., Small Anim. Pract. 22:645-682.

Tarvin G.B. \& Lenehan T.M. 1996. Osteotomia pélvica, p.622-627. In: Bojrab J.M. (Ed.), Técnicas Atuais em Cirurgia de Pequenos Animais. $3^{\text {a }}$ ed. Roca, São Paulo.

Tomlinson J.L. \& Cook J.L. 2002. Effects of degree of acetabular rotation after triple pelvic osteotomy on the position of the femoral head in relationship to the acetabulum. Vet. Surg. 31:398-403.

Tomlinson J.L. \& Johnson J.A. 2000. Quantification of measurement of femoral head coverage and Norberg angle within and among four breeds of dogs. Am. J. Vet. Res. 61:1492-1500.

Whelan M.F., McCarthy R.J., Boudrieau R.J. \& Kraus K.H. 2004. Increase sacral screw purchase minimizes screw loosening in canine triple pelvic osteotomy. Vet. Surg. 33:609-614. 